Check for updates

London, UK

Cite this as: BMJ 2021;374:n2079 http://dx.doi.org/10.1136/bmi.n2079 Published: 20 August 2021

\section{Hospital bed occupancy rates in England reach dangerously high levels}

\section{Adrian O'Dowd}

The number of beds occupied in hospitals in England is nearing levels seen before the covid-19 pandemic, according to new data.

Overall, $83.8 \%$ of the 123707 beds available overnight were occupied between April and June this year, show bed occupancy rates data ${ }^{1}$ from NHS England.

More worryingly, 82 trusts exceeded the $85 \%$ rate which is generally considered to be the limit at which hospitals are able to work safely and effectively. Moreover, 35 of these trusts had reached occupancy levels above $90 \%$, while six trusts reached levels above $95 \%$.

NHS England cautioned that in order to treat covid and non-covid patients separately and to meet enhanced infection prevention control measures, hospital capacity has had to be organised in new ways as a result of the pandemic. This has meant that beds and staff in emergency and elective settings within hospitals have had to be deployed differently from previous years and, in general, "hospitals will experience capacity pressures at lower overall occupancy rates than would previously have been the case," according to NHS England.

Nevertheless, the data show that the bed occupancy rate of $83.8 \%$ for the first quarter of 2021-22 was nearly as high as the $88.2 \%$ rate recorded for the same quarter in $2019-20$, before the pandemic.

Occupancy rates for the same quarter in the previous two years (2018-19 and 2017-18) were $87.8 \%$ and $87.2 \%$ respectively, showing there has been an ongoing trend of high occupancy rates for the past few years.

NHS Providers, the membership organisation for NHS hospital, mental health, community, and ambulance services, said the latest figures highlighted the scale of current pressure across the whole health service.

Its chief executive, Chris Hopson, said, "Evidence clearly shows that hospitals work most safely and effectively at bed occupancy levels no higher than $85 \%$. It is therefore concerning that today's figures show that 82 trusts exceeded this over the past quarter with 35 of these trusts experiencing bed occupancy levels above $90 \%$ and six reaching over $95 \%$.

"High bed occupancy levels have a direct impact on trusts' ability to provide safe and timely services for patients."

Even though the number of beds available was slowly increasing, he added, the NHS had lost more than 4500 general and acute beds compared with before the pandemic, because of the need for stringent covid-19 infection control measures, which was adding to pressures on the service.

"Treating patients quickly and effectively depends on having the right number of beds," said Hopson. "We need the NHS to be resourced properly which is why it's so important the service gets the immediate funding it needs for the second half of this financial year and in the upcoming comprehensive spending review."

NHS Providers said it anticipated that bed occupancy figures for the next quarter (July to September) of this year would be even higher because several trust chief executives had told them that their hospitals were busier than they had ever been over the summer.

NHS England. Bed availability and occupancy data: overnight. 19 August 2021. www.england.nhs.uk/statistics/statistical-work-areas/bed-availability-and-occupancy. 